\title{
Análisis de la ampliación de cobertura a la categoría de trabajadoras del hogar en la mejora de la calidad de servicio del afiliado al IESS del cantón Milagro del periodo 2015-17.
}

\begin{abstract}
Analysis of the extension of coverage to the category of domestic workers in the improvement of the service quality of the affiliate to the IESS of the canton Milagro of the period 2015-17.
\end{abstract}

Mayorga Arias Miriam Paola ${ }^{1}, \&$ Gamboa Poveda Jinsop Elias. ${ }^{2}$

DOI: $\underline{\text { https://doi.org/10.33262/visionariodigital.v3i2.399 }}$

\section{Resumen.}

La seguridad social es una de las preocupaciones más urgentes para los ecuatorianos en la actualidad. Sin embargo, carecemos de un sistema eficaz y completo, que abarque todos los renglones a proteger y a todos los grupos humanos y, en el los sectores donde están implementados, son insuficientes los mecanismos de acceso y existen falencias que provocan desatención y demora, pero, sobre todo, el índice de afiliación es aún bajo, debido a la inexistencia de recursos y la falta de comunicación empresarial y preparación del personal para la atención a los usuarios y posibles usuarios del servicio. La Ley debe prever los mecanismos adecuados para su implementación y calidad. Para la mejora continua del proceso de afiliación y calidad del servicio se debe implementar mecanismos adecuados de medición del impacto de la Ley actual y capacitar adecuadamente al personal existente en el sistema de atención y en especial en los hospitales. Nuestro objetivo es analizar y compararlos datos de afiliación y satisfacción del cliente en el periodo 2015 - 2017 y proponer soluciones en caso de resultar bajos los resultados. Se elaborará un modelo con los datos para evaluar la variabilidad de afiliación al IESS de trabajadoras del hogar. La implementación de nuestro modelo permitirá contar con un instrumento de medición de tendencias que permita tomar decisiones oportunas que mejoren el indicador de afiliación y la satisfacción de los usuarios del servicio.

Palabras claves: Análisis, Coberturas, calidad del servicio, IESS, trabajadoras del hogar.

\footnotetext{
1 Instituto Ecuatoriano de Seguridad Social, Milagro, Ecuador, cym14f@hotmail.com , mmayorgaa@iess.gob.ec

2 Universidad Estatal de Milagro, Milagro, Ecuador, jgamboap@unemi.edu.ec
} 


\begin{abstract}
.
Social security is one of the most urgent concerns for Ecuadorians today. However, we lack an effective and comprehensive system that covers all the lines to be protected and all human groups and, in the sectors where they are implemented, access mechanisms are insufficient and there are shortcomings that cause inattention and delay, but, Above all, the affiliation index is still low, due to the lack of resources and the lack of business communication and preparation of the staff to serve users and potential users of the service. The Law must provide adequate mechanisms for its implementation and quality. For the continuous improvement of the affiliation process and quality of service, adequate mechanisms should be implemented to measure the impact of the current Law and adequately train existing personnel in the care system and especially in hospitals. Our objective is to analyze and compare affiliation and customer satisfaction data in the period 2015 - 2017 and propose solutions if the results are low. A model with the data will be developed to evaluate the variability of IESS affiliation of domestic workers. The implementation of our model will allow us to have a trend measurement instrument that allows us to take timely decisions that improve the affiliation indicator and the satisfaction of the users of the service.
\end{abstract}

Keywords: Analysis, coverage, quality of service, IESS, domestic workers.

\title{
Introducción
}

El presente artículo hace referencia al Instituto de Seguridad Social del Ecuador que es un ente encargado en ofrecer servicios médicos y de salud a todos los afiliados de acuerdo a la provincia o ciudad en la que se encuentren, este instituto está regulado por el Gobierno Central y sus reformas y estatutos se rigen por las leyes vigentes en el país.

A partir del 2015 entró en vigencia la afiliación para las trabajadoras del hogar, por lo que este número pudiera inferir en más carga laboral para los funcionarios médicos y administrativos, por lo que surge la necesidad de una investigación para identificar oportunidades de mejora en la inclusión de este tipo de trabajo.

Esta investigación busca inicialmente cuantificar el impacto de la Ley establecida en el 2015 para las trabajadoras del hogar, así como mejorar el servicio que reciben, esto con la ayuda de encuestas e indicadores de satisfacción, además podremos identificar las áreas de salud de mayor demanda y los motivos por los cuales se acercan al Hospital IESS del cantón Milagro.

La misma se llevó a cabo en el Hospital de Milagro por lo que la información obtenida servirá para que exista una voz de alerta de las situaciones por las que estas personas asisten y analizar su cobertura tanto para tiempo parcial como por tiempo completo para demostrar si tiene un impacto positivo o negativo en el incremento de afiliados. Asimismo, si el incremento o disminución tiene algún efecto en el trabajo diario del personal del Hospital. 
La investigación se llevó a cabo en el cantón Milagro de la provincia de Guayas, analizando la incorporación de las trabajadoras del hogar al IESS, durante el periodo 2015 - 2017 y la incidencia del Hospital del IESS de la localidad en sus resultados, a partir de la preparación del personal, la calidad de los servicios y la divulgación de sus beneficios. Se tomó como población la totalidad de las trabajadoras del hogar del cantón y como muestra las afiliadas al IESS a partir de la promulgación de la Ley.

\section{Desarrollo.}

\section{Metodología.}

La investigación a realizarse fue cuanti-cualitativa, ya que se analizó estadísticamente las cifras de afiliación al IESS en el cantón Milagro de la provincia de Guayas y se determinarán las falencias en el sistema de calidad del Hospital del IESS de Milagro. Asimismo, cualitativamente se revisó como observación directa el comportamiento de los afiliados ante, durante y al final de la consulta y del ingreso a las instalaciones del Hospital para poder registrar el ciclo completo de sus actividades y poder segmentarlo.

Objetivamente, se realizó una encuesta a los funcionarios administrativos, médicos y usuarios en general para medir el nivel de servicio percibido y tomar en consideración la información que puedan proporcionar de casos en los que hayan sentido vulnerados sus derechos como afiliados al Instituto de Seguridad Social ubicado en el Cantón Milagro.

\subsubsection{Los métodos de investigación a utilizar serán:}

Estadístico: para la recolección de los datos referidos a la incorporación o afiliación y de las encuestas que se realicen.

Inductivo - deductivo: para determinar las causas y consecuencias del objeto de estudio analizado y llegar a conclusiones.

Analítico: Para realizar la evaluación de los resultados alcanzados y junto con la observación directa poder determinar si existe uno o varios casos en los que se pueda mejorar.

Sintético: Para resumir los resultados de la medición de las variables.

Histórico - comparativo: Para sistematizar los conocimientos existentes acerca del objeto de estudio y comparar los indicadores que permitan arribar a conclusiones adecuadas desde el punto de vista científico.

Se trabajará con una amplia población de la totalidad de los habitantes del cantón Milagro y con una muestra representativa, tomada de los censos correspondientes donde se establezcan los afiliados por meses y bienio y se mida la calidad del servicio.

Se elaborarán entrevistas a funcionarios, especialistas y usuarios del sistema de seguridad social del cantón y el hospital de Milagro. 
Cantidad: 200 Encuestas

Entrevistas dirigidas a:

- 50 funcionarios Administrativos

- 50 funcionarios médicos

- 100 usuarios del Sistemas

\subsubsection{Encuesta:}

De acuerdo su satisfacción con los servicios ofertados por el Hospital General de Milagro, por favor coloque una $(X)$ en el casillero que más le define:

1. Calidad en la atención y Call Center

\begin{tabular}{|l|l|l|l|l|}
\hline Excelente & Muy Bueno & Bueno & Regular & Insuficiente \\
\hline & & & & \\
\hline
\end{tabular}

Esta pregunta con el fin de evaluar la percepción de calidad que tienen los afiliados con respecto a la atención brindada durante su visita al Hospital y por medio del Call Center.

2. Disponibilidad de Medicamentos

\begin{tabular}{|l|l|l|l|l|}
\hline Excelente & Muy Bueno & Bueno & Regular & Insuficiente \\
\hline & & & & \\
\hline
\end{tabular}

Es necesario conocer la disponibilidad de medicamentos, no sólo mediante la planificación y abastecimiento sino a la entrega, los afiliados son quienes concluyen el final de este flujo de disponibilidad de medicamentos.

3. Disponibilidad de citas médicas

\begin{tabular}{|l|l|l|l|l|}
\hline Excelente & Muy Bueno & Bueno & Regular & Insuficiente \\
\hline & & & & \\
\hline
\end{tabular}

De acuerdo a los registros estadísticos se encuentra la cantidad de afiliados atendidos, pero es necesario también la cantidad de asegurados que sientes que sus citas son al tiempo que la necesitan o que las pueden conseguir de manera efectiva.

4. Infraestructura y equipamiento del hospital

\begin{tabular}{|l|c|c|c|c|}
\hline Excelente & $\begin{array}{c}\text { Muy } \\
\text { Bueno }\end{array}$ & Bueno & Regular & Insuficiente \\
\hline & & & & \\
\hline
\end{tabular}


Para este capítulo es primordial que se pueda desarrollar cada uno de los objetivos antes señalados, por lo que los resultados arrojados durante las encuestas, la observación directa y las investigaciones realizadas detallan lo siguiente de acuerdo a lo antes expuesto en el capítulo uno de este trabajo de investigación. Estos resultados se generaron del análisis de las encuestas, de la investigación de documentos, diarios, leyes y de información del Hospital General de Milagro con fines educativos para poder obtener los resultados que se mostraran a continuación.

Dentro del primer objetivo propuesto en este trabajo de investigación está el realizar un análisis de las coberturas que tienen las trabajadoras del hogar al estar afiliadas tiempo parcial y tiempo completo en la mejora de la calidad del Servicio del Afiliado al IESS del Cantón Milagro en el periodo 2015 al 2017. A continuación, se detallan las coberturas que tienen las trabajadoras del hogar: Afiliación, Salud, riesgos del trabajo, auxilio de funerales. Por lo general los afiliados cuentan con 8 prestaciones, pero las trabajadoras del hogar forman parte de los trabajadores que su tiempo se cataloga como parcial.

"El Instituto Ecuatoriano de Seguridad Social (IESS) se enfocará en ocho prestaciones estratégicas, una vez que entre a operar su banco. Estas son: afiliación, jubilación, salud, riesgos del trabajo, seguro campesino, cesantía, auxilio de funerales y fondos de reserva." (Comercio, 2015)

Este grupo de trabajadoras al recibir la cobertura de salud tiene derecho a la atención médica en general como un afiliado de tiempo completo por lo que aumentó la demanda de médicos, medicina, consultorios y tiempos de espera entre un afiliado y otro provocando una disminución importante de la calidad, esto está detallado más adelante en la percepción de los afiliados, médicos y funcionarios del Hospital General de Milagro.

El segundo objetivo está ligado directamente al primero ya que toma en consideración la cantidad de personas atendidas como los recursos con los que cuenta el hospital, por lo que se muestra a continuación indicadores estadísticos de los años en referencia del estudio para determinar el incremento de afiliados versus el mantener la misma cantidad de recursos, lo que causó indiscutiblemente problemas de calidad en la atención y en la forma de tomar decisiones administrativas que beneficien a la población afiliada en general y a los médicos y funcionarios que atienden a estas personas.

Productividad por Atención médica 2015 - 2016 - 2017

Entre las 21 especialidades que ofrece el Seguro social están las siguientes:

Calificación médica, cirugía general, cirugía pediátrica, dermatología, endocrinología, fisiatría, gastroenterología, ginecología, medicina general, medicina interna, nutrición, otorrinolaringología, psicología, psiquiatría, pediatría, medicina a domicilio, traumatología, urología, cardiología, neumología, medicina preventiva.

Cada año la cantidad de médicos varía, en consecuencia, hay menos recursos para atender a los afiliados siendo este un factor clave para mantener la atención y la calidad de los servicios, ya que debe ser equitativa la cantidad de pacientes de acuerdo a la especialidad y el tipo de caso que se atiende. En el año 2015, al iniciar la afiliación de las trabajadoras del hogar se nota un volumen mayor que en años posteriores, pero también mayor cantidad de médicos, un dato relevante es que en este año los médicos cubanos llegaron el país por convenio en el Ecuador, el siguiente año 
la cantidad de médicos disminuye ofreciendo menos plazas de atención y en 2017 aumentan 3 médicos y se nota el aumento de la productividad y atención.

\begin{tabular}{|l|c|c|c|}
\hline \multicolumn{1}{|c|}{ Especialidades } & $\mathbf{2 0 1 5}$ & $\mathbf{2 0 1 6}$ & $\mathbf{2 0 1 7}$ \\
\hline Cantidad de Médicos & 56 & 50 & 53 \\
\hline 21 especialidades & 191033 & 165284 & 168480 \\
\hline
\end{tabular}

Elaborado por: Autores.

Fuente: Autores.

*Para más detalle se puede revisar el anexo 2 de este documento.

El tercer objetivo tiene que ver con la atención percibida por las trabajadoras del hogar en el área de consulta externa por parte del personal del Hospital para la mejora de la calidad del servicio del Afiliado en el Iess del Cantón Milagro en el periodo señalado en este estudio. A continuación, se muestran los resultados de las encuestas realizadas a 200 personas como se detalla en el capítulo 3 de este proyecto de investigación:

1. Calidad en la atención y Call Center

\begin{tabular}{|c|r|}
\hline Excelente & $10,50 \%$ \\
\hline Muy Bueno & $7,00 \%$ \\
\hline Bueno & $33,50 \%$ \\
\hline Regular & $30,50 \%$ \\
\hline Insuficiente & $18,50 \%$ \\
\hline
\end{tabular}

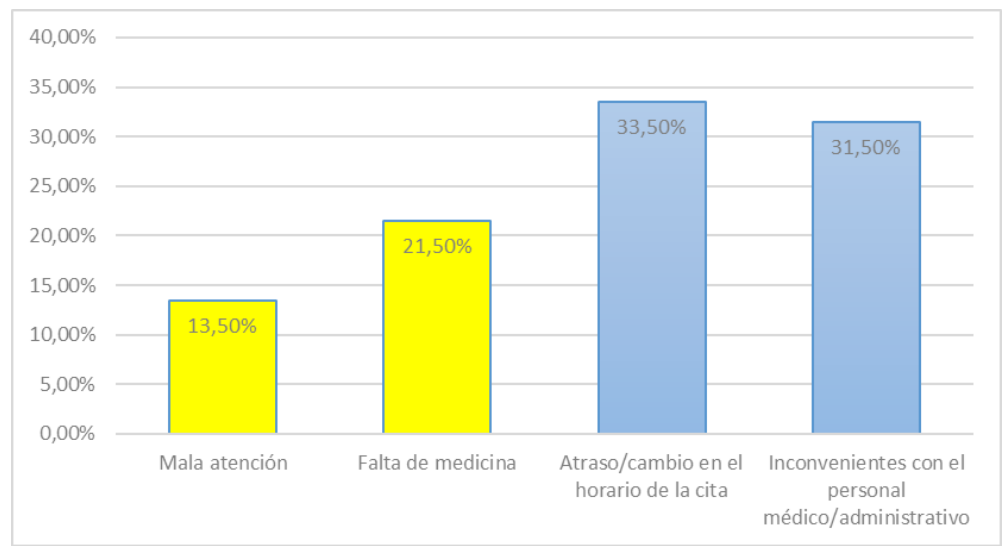

Elaborado por: Autores.

El 33,5\% de los encuestados menciona que la calidad en la atención y el call center es bueno, el $7 \%$ muy bueno, el 30,5\% que es regular, el 10,5\% que es excelente y el 18,50\% que es insuficiente siendo este grupo el que tiene malas experiencias con citas anteriores 
o mediante el Call Center. El 17,5\% reconoce que la atención está entre muy buena y excelente.

2. Disponibilidad de Medicamentos.

\begin{tabular}{|c|r|}
\hline Excelente & $10,50 \%$ \\
\hline Muy Bueno & $17,00 \%$ \\
\hline Bueno & $39,50 \%$ \\
\hline Regular & $24,50 \%$ \\
\hline Insuficiente & $8,50 \%$ \\
\hline
\end{tabular}

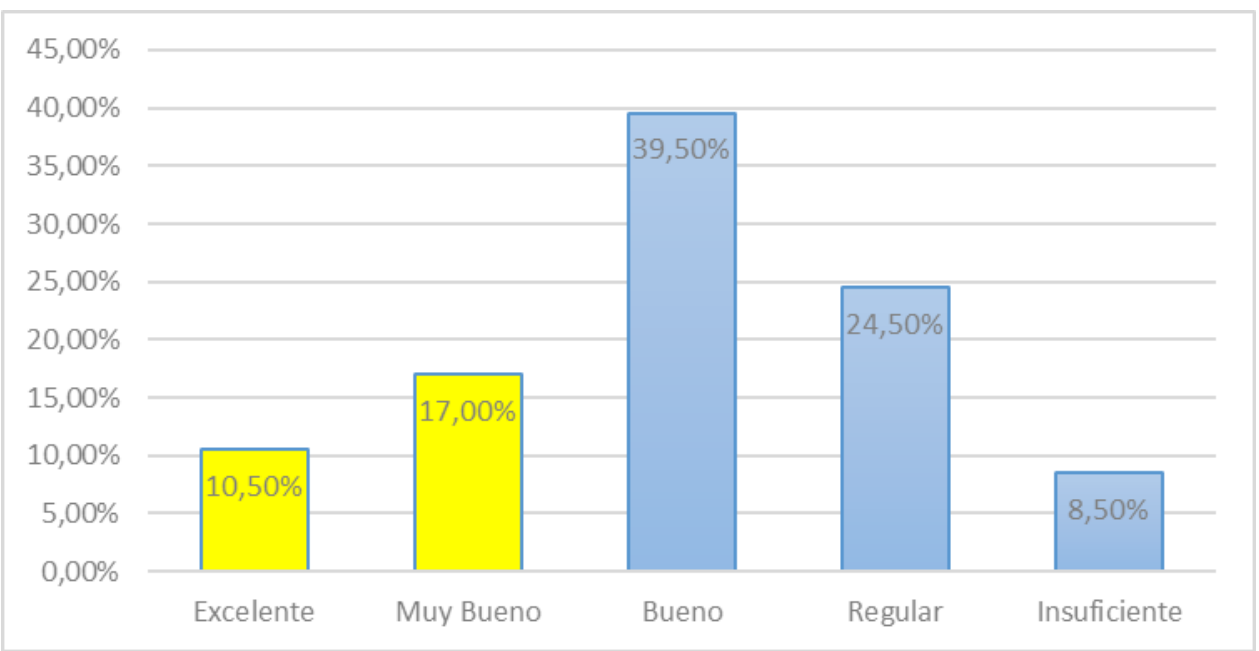

Elaborado por: Autores.

Con respecto a la disponibilidad de los medicamentos, de las personas encuestadas el $39,5 \%$ menciona que es bueno, el $24,5 \%$ que es regular, el $17 \%$ que es muy bueno, el $10,5 \%$ que es excelente y hay un $8,5 \%$ que considera que es insuficiente. El 27,5\% considera que está entre muy bueno y excelente. En general consideran que las medicinas más costosas e importantes por lo general no se encuentran disponibles y que esto debería mejorar.

3. Disponibilidad de citas médicas.

\begin{tabular}{|c|r|}
\hline Excelente & $21,50 \%$ \\
\hline Muy Bueno & $18,00 \%$ \\
\hline Bueno & $26,50 \%$ \\
\hline Regular & $24,50 \%$ \\
\hline Insuficiente & $9,50 \%$ \\
\hline
\end{tabular}




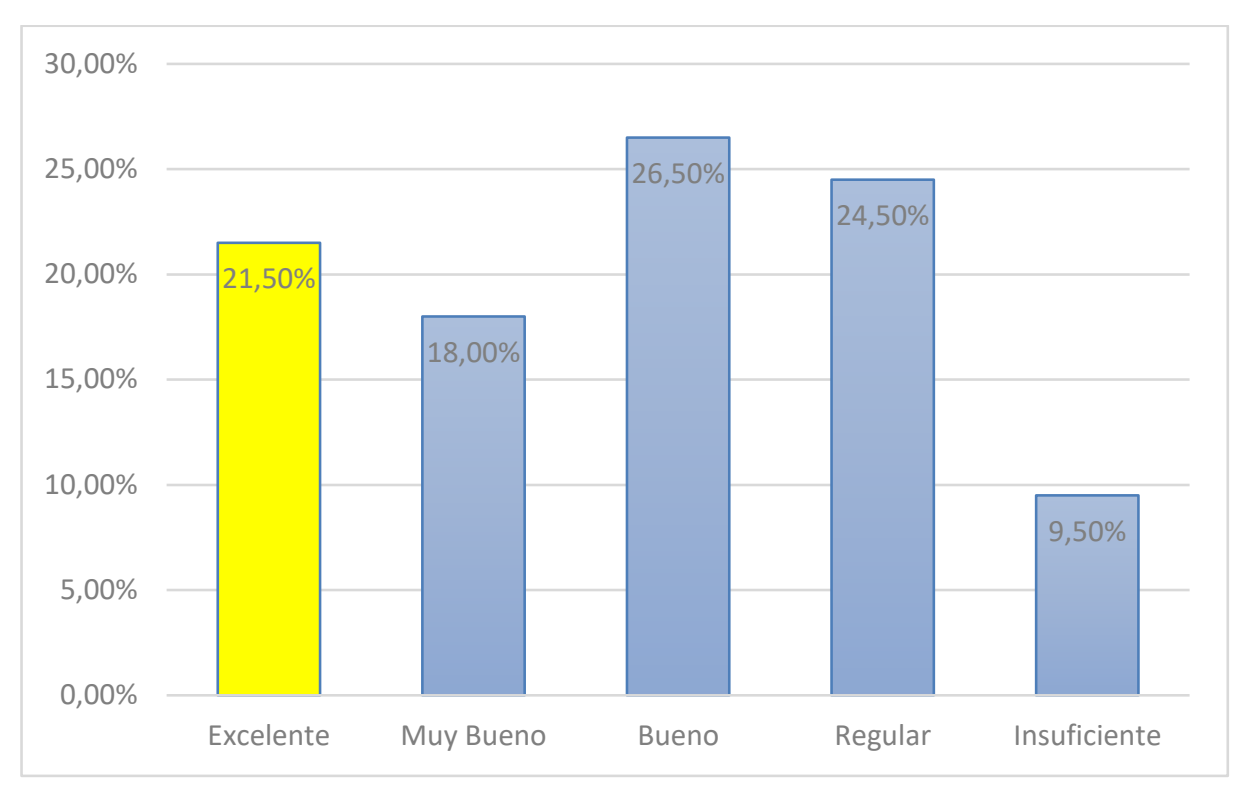

Elaborado por: la autora

Con respecto a las citas médicas, del total de encuestados, el 34\% considera entre insuficiente e irregular la disponibilidad de citas médicas, mientras que otro $26,5 \%$ considera que es bueno, un $18 \%$ que muy bueno y un $21,5 \%$ que es excelente. Este último grupo menciona que ha mejorado mucho la disponibilidad de citas y que facilita a las personas que pueden conseguir cita un fin de semana.

4. Infraestructura y equipamiento del hospital.

\begin{tabular}{|c|r|}
\hline Excelente & $18,00 \%$ \\
\hline Muy Bueno & $26,00 \%$ \\
\hline Bueno & $28,00 \%$ \\
\hline Regular & $24,50 \%$ \\
\hline Insuficiente & $3,50 \%$ \\
\hline
\end{tabular}

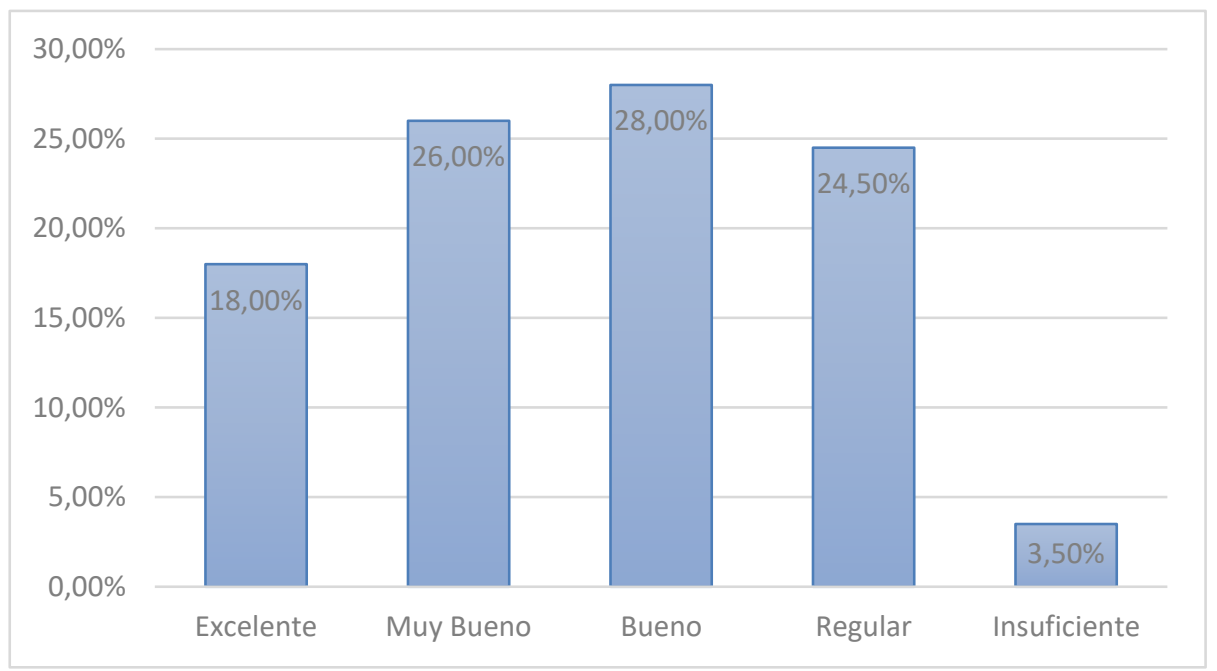

Elaborado por: la autora 
De acuerdo a los resultados arrojados de las encuestas, se muestran los siguientes resultados con respecto a lo que los afiliados perciben de la infraestructura y equipamiento del hospital, el 28\% considera que está entre regular e insuficiente, el $28 \%$ considera que es bueno y el $44 \%$ considera que es entre muy bueno y excelente, este grupo considera que con el tiempo se han implementado nuevas áreas y máquinas para exámenes médicos y que eso es un plus para sentir la calidad en el hospital.

1. ¿Ha tenido algún inconveniente no resuelto con el Hospital general de Milagro, sus colaboradores o la atención que se ofrece?

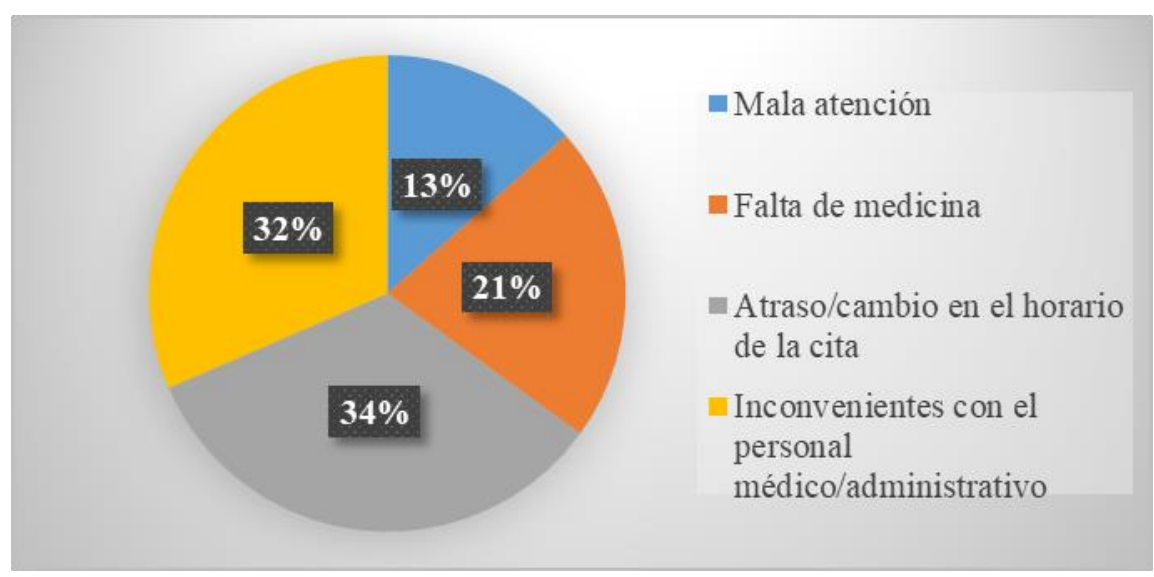

Elaborado por: La autora.

Los resultados arrojados por las encuestas realizadas mencionan que entre los inconvenientes que han tenido los afiliados y que han percibido los funcionarios y personal médico están los siguientes: $34 \%$ en atrasos o cambios en el horario de las citas perjudicando el tiempo invertido para asistir a la misma, el $32 \%$ ha tenido inconvenientes con el personal médico o administrativo por temas dela consulta, el $21 \%$ por falta de medicina y refutando que les descuentan un valor justamente para que esté abastecido el Hospital de insumos y medicinas necesarias y finalmente el $13 \%$ menciona una mala atención recibida por cualquier persona que forme parte del Hospital IESS del cantón Milagro.

El cuarto objetivo que es el de proponer mejoras en el servicio de las trabajadoras del hogar afiliadas al IESS en el Cantón Milagro, que permitan optimizar sus coberturas en la mejora de la calidad del Servicio del Afiliado en el periodo mencionado al inicio de este trabajo de investigación.

\section{Discusión.}

En la época actual al estimar que la población ecuatoriana sostiene un ritmo de crecimiento acelerado, que demanda la existencia de un sistema de salud universal y eficiente, que permita la cobertura del total de los habitantes, y especialmente de los grupos etarios y sociales más vulnerables. 
El constante aumento del número de trabajadores informales, el alto índice de nacimientos y la disminución de los fallecimientos hace que sea mayor el número de personas no afiliadas que el de afiliados al IESS.Y al tomarse en cuenta, la carencia de un análisis de la atención que reciben las trabajadoras del Hogar en el área de emergencia y consulta externa tiene cegada la toma de decisiones para estar al corriente del tipo de cobertura que más utilizan o como se puede mejorar. Finalmente, la falta de estos análisis ha creado un espacio significativo que hace desconocer a la alta gerencia el incremento o que tan favorable fue la nueva ley en el sector de la salud. La desventaja principal que existe hoy en día es la falta de información acerca de satisfacción y servicios que reciben estas trabajadoras del hogar. Además de esto, se desconoce si los empleadores están cumpliendo o no con lo dispuesto o si han tomado otras medidas para no cumplir con esta disposición.

Se busca analizar la ampliación de la cobertura a la categoría de trabajadoras del hogar, este análisis servirá para medir la afiliación en tiempo parcial y completo de las trabajadoras del hogar. Asimismo, se evaluará el impacto que generó en cantidad de personas atendidas y los recursos utilizados con la finalidad de obtener propuestas para las mejoras en servicio.

¿Qué se entiende por trabajadoras del hogar?

¿Existe un compromiso del IESS con la mejora y ampliación del servicio a sus afiliados del cantón Milagro del periodo 2015-2017?

¿Existe una relación entre la ampliación de cobertura a la categoría de trabajadoras del hogar en la mejora de la calidad de servicio del afiliado al IESS del cantón Milagro del periodo 2015-2017?

¿Es factible la mejora de la calidad de servicio del afiliado al IESS del cantón Milagro del periodo 2015-2017, a partir del aumento de la afiliación de las trabajadoras del hogar?

En la presente investigación se deberá conocer a cabalidad la fundamentación teórica en referencia a:

Conveniencia: Es necesaria la investigación ya que nos permitirá evaluar adecuadamente los índices de afiliación y calidad del servicio brindado a los trabajadores no remunerados del hogar a partir de la puesta en vigor de la Ley.

Relevancia social: Nuestra investigación intenta demostrar si se capacita adecuadamente al personal de servicios hospitalarios, se informa a la población y se aplican los instrumentos de medición correctos, se logrará una mayor incorporación de los trabajadores no remunerados del hogar a los servicios del IESS. De esta manera se beneficiará un sector importante de la población que constituye un alto por ciento de los ecuatorianos de bajos ingresos.

Implicaciones prácticas: La puesta en práctica del sistema permitirá medir el índice de incorporación y los estándares de calidad del servicio del IESS en el cantón Milagro de 
la provincia de Guayas, lo que se podrá hacer extensivo al resto del país, de demostrarse su viabilidad.

Valor teórico: Con la investigación se logrará llenar un vacío en el mecanismo de evaluación de indicadores importantes y se podrán generalizar estos instrumentos a otras regiones del país.

Utilidad metodológica: La investigación permitirá crear un nuevo instrumento para recolectar y analizar los datos.

Así como también la conceptualización de lo que la calidad del servicio que tipos de modelos se encuentran para poder determinar la misma en las actividades de un centro de salud.

Para Grönroos (1990) afirma que cualquier producto físico puede convertirse en un servicio para un cliente si la única persona que vende el servicio proporcionado el servicio se convierte en una solución para satisfacer la demanda de un cliente.

Blois (1974) señala que los servicios son actividades ofrecidas en venta que para el comprador presenta beneficios y satisfacción.

Stanton (1974) define los servicios como actividades intangibles. que proporcionan satisfacción cuando se comercializan a consumidores que no están vinculados a la venta de un producto u otro servicio.

Como hay una gran cantidad de definiciones hechas sobre el servicio en toda la literatura, Grönroos (1990) se combinó a partir de varias definiciones que son más identificables: "Un servicio es una actividad o serie de actividades de naturaleza más o menos intangible que normalmente, pero no necesariamente, tiene lugar en las interacciones entre el cliente y los empleados del servicio y / o física recursos o bienes y / o sistemas del proveedor de servicios, que se proporcionan como soluciones para el cliente.

La calidad también se ha definido de muchas maneras diferentes en muchas literaturas diferentes.

De acuerdo a Juran (1988) identificó la calidad como un atributo que se relaciona con el cliente. Más Interesante es en qué perspectiva se define la calidad. Por ejemplo, Grönroos (1990). define la calidad como lo que el cliente percibe que es donde Juran (1988) define la calidad como las características de los productos o servicios que satisfacen las expectativas de clientes y llevan a la satisfacción del cliente.

La naturaleza del servicio se define como una actividad que involucra un producto y se proporciona como una solución para satisfacer la demanda de un cliente y esa calidad es el resultado de estar satisfecho o no después de haber evaluado si un servicio, así como la prestación del servicio cumplió con las expectativas de un cliente.

Sin embargo, esto se considera una percepción de la persona que recibe el servicio y la calidad depende entonces de si el servicio satisface las necesidades, deseos y expectativas de ese servicio con clientes específicos. Dentro de esta historia, la calidad del servicio es vista como la evaluación total de qué tan bien un servicio prestado cumple con las 
expectativas del cliente. (Zeithaml, Berry \& Parasuraman 1988) \& (Parasuraman, Zeithaml \& Berry, 1988).

Esto a su vez da como resultado la satisfacción del cliente o no, y es esta satisfacción o no lo que determina la calidad del servicio para el negocio según lo percibido por el cliente.

El hecho de que los clientes estén satisfechos o no por los servicios prestados determina cómo el negocio funciona bien según lo percibe el cliente y esta información se utiliza para mejorar los servicios, identificar problemas y evaluar mejor la satisfacción del cliente.

A la luz de las numerosas declaraciones sobre el tema, una definición propia de la calidad del servicio es propuesto, a saber; La calidad del servicio es el resultado de la relación entre el cliente expectativas que se derivan antes de la entrega del servicio frente a la experiencia del cliente que se produce la prestación de servicios que es altamente dependiente del rendimiento, la actitud y el comportamiento de un empleado de contacto guiado por la participación de la gerencia en términos de formación, motivaciones, liderazgo y compromiso.

\section{Concepción actual del trabajo no remunerado del hogar y el servicio doméstico.}

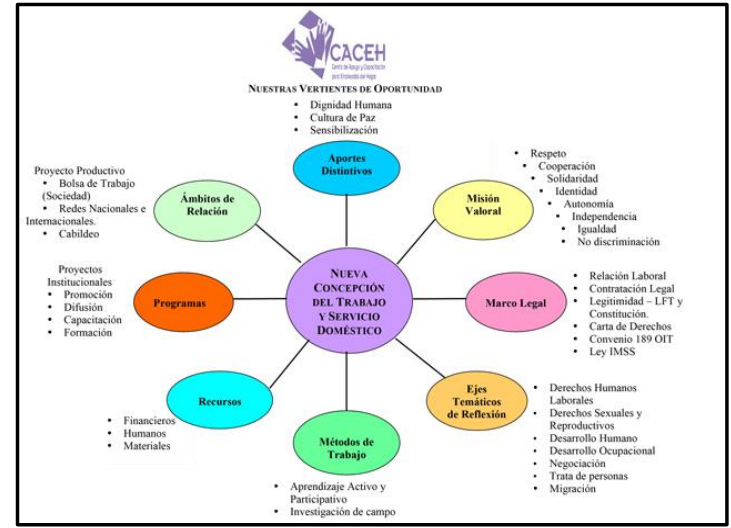

Fuente: www.caceh.org.mx/

\section{Evaluación unidimensional de la calidad del servicio}

En referencia a la calidad que se recibe en el servicio permite la evaluación de manera global, en base a la excelencia que se observa en la misma, en el desempeño laboral del personal a cargo, sin tomar en cuenta los atributos específicos en el servicio.

Al desarrollar una evaluación global, esta da facilidad a que se tenga que implementar varios modelos causales lo que da como resultado que se tenga una caracterización en la calidad que es recibida, se puede además analizar tanto la fiabilidad y validez, pero no es recomendable para analizar los atributos específicos del servicio, es por ello que se indica desde una perspectiva directiva que esta puede resultar no tan agradable su utilización. 
De acuerdo a lo que se indica por parte de Martinez y Martinez (2007), afirman que la medida de un ítem se comporta de forma prácticamente idéntica que la escala multidimensional en relación a los intervalos de confianza de los parámetros.

Se han encontrado interesantes resultados comparados con las realizadas con múltiples indicadores (Bergkvist y Rossiter, 2007; Martínez y Martínez, 2010).

Este tipo de evaluación global y unidimensional de la calidad percibida del servicio resulta económico y fácil para la aplicación de encuestas y es también útil para correlacionar este indicador con otros asociados al servicio, o con otros resultados de la organización, como por ejemplo: correlacionar calidad global percibida del servicio y la eficiencia del mismo (Torres y Vásquez, 2011).

Cronología sobre las principales propuestas teóricas sobre calidad del servicio, su evaluación y hechos internacionales relevantes

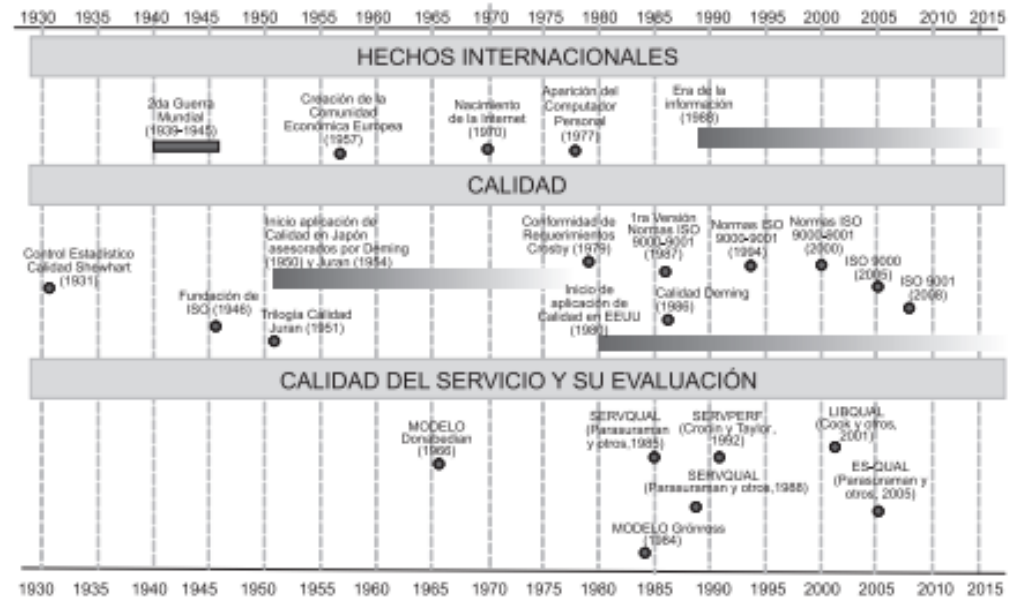

Fuente: Elaboración Propia

\section{Modelo de calidad de la atención médica de Donabedian}

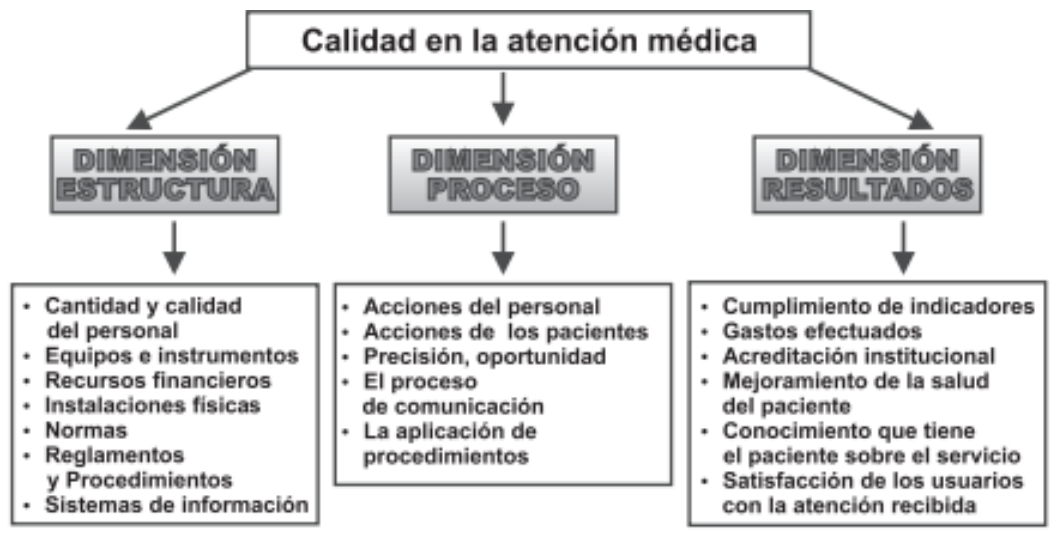

Elaboración propia.

Fuente: Donabedian (1966) 


\section{Dimensiones y atributos del SERVQUAL}

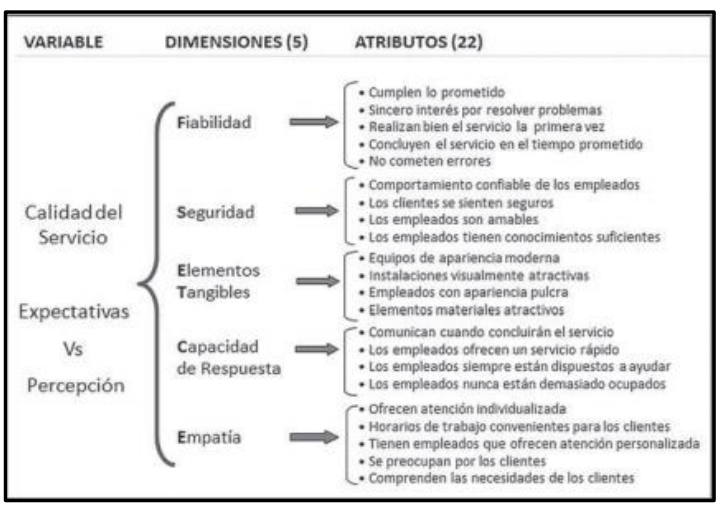

Elaboración propia.

Fuente: Parasuraman y otros (1988).

\section{Modelo Jerárquico Multidimensional de Brady y Cronin}

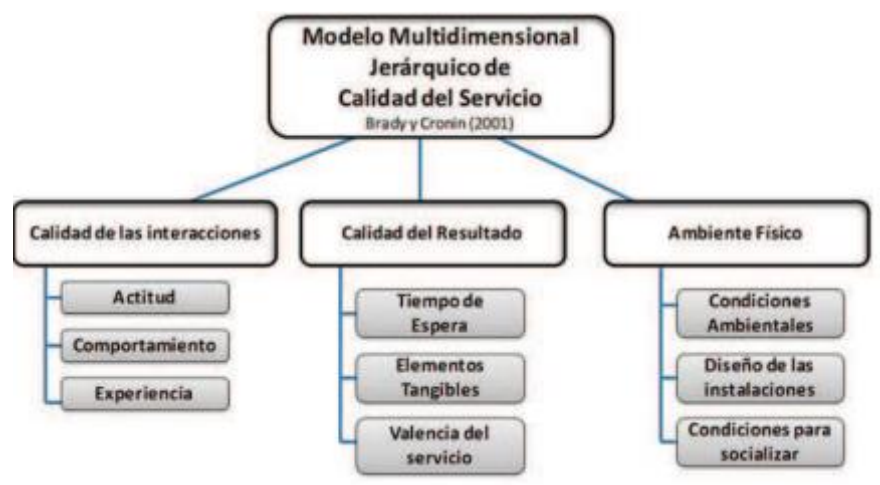

Elaboración propia.

Fuente: Brady y Cronin (2001).

Modelo de calidad del servicio en bibliotecas LibQUAL

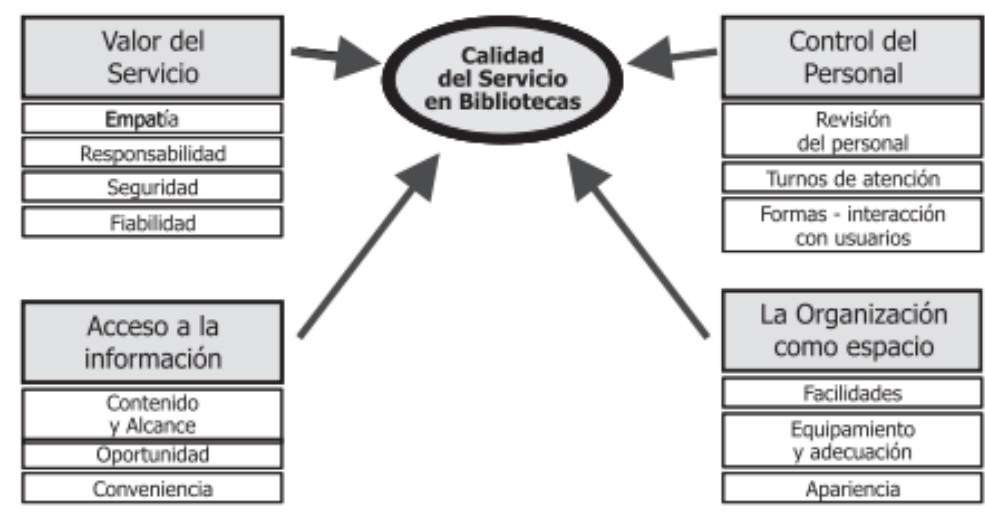

Elaboración propia.

Fuente: Játiva y Gallo (2006). 


\section{Conclusión.}

- En primer lugar, se concluyó que la calidad del servicio, tal como la perciben los clientes, es el resultado de lo bien que el servicio coincide con las expectativas. En segundo lugar, que la calidad del servicio era determinado por dos dimensiones, a saber; Expectativas y experiencia y cada uno de estos.

- Las dimensiones tenían factores subyacentes. En tercer lugar, 5 factores determinaron el resultado de Calidad del servicio que utilizan los clientes para completar el juicio durante el servicio. entrega, a saber; Tangibles, fiabilidad, capacidad de respuesta, seguridad y empatía.

- Como conclusión se puede mencionar además que El producto es lo que es, en nuestro caso muy excelente. Y el cliente obtiene lo que espera como el producto es lo que es, pero la variable principal es el personal y cómo el cliente recibe el Servicio.

- En referencia a los modelos y su aplicación en lo que concierne a la atención del servicio se evidencia la preponderancia del modelo SERVQUAL para medir la calidad, para lo cual se realizar en base a las diversas técnicas que ofrece dicho modelo con ello se establecerá la calidad que existe en un servicio.

\section{Referencia Bibliográfica.}

AJAM, MEHDI; JAMIL SADEGHIFAR, MINA ANJOMSHOA, SAEID MAHMOUDI, HASAN HONARVAR and SEYYED MOUSAVI (2014). Assessing Quality of Healthcare Service by the SERVQUAL Model: A Case Study of a Field Hospital. Journal of Military Medicine.Vol. 15. No 4. pp. 273-279. ALNSOUR,

MUHAMMED; BANDAR ABU TAYEH and MOHAMMED AWWAD ALZYADAT (2014). Using SERVQUAL to assess the quality of service provided by Jordanian telecommunications Sector. International Journal of Commerce and Management. Vol. 24. No. 3. pp. 209 - 218.

Alcalá, G. (2007). El clima de una organización. Santa fé.

Asamblea Nacional. (13 de julio de 2011). Constitución de la República 2008. Obtenido de Asamblea Nacional: www.oas.org/juridico/PDFs/mesicic4_ecu_const.pdf

Asamblea Nacional. (13 de junio de 2011). www.oas.org/juridico/PDFs/mesicic4_ecu_const.pdf. Obtenido de Constitución 2008: www.oas.org/juridico/PDFs/mesicic4_ecu_const.pdf

BERGKVIST, LARS and JOHN ROSSITER (2007). The predictive validity of multipleitem versus single-item measures of the same construct. Journal of Marketing Research. No 44. pp. 175-184.

BRADY, MICHAEL and CRONIN JOSEPH (2001). Some new thoughts on conceptualizing perceived service quality: A hierarchical approach. Journal of Marketing. Vol. 65. No 3. pp. 34-49.

Bonta, P. (s.f.). En M. Farber, 199 preguntas sobre Marketing y Publicidad.

CONSTITUCION DE LA REPUBLICA DEL ECUADOR 2008. (s.f.). 
COOK, COLLEEN; HEATH FRED and BRUCE THOMPSON (2001). The search for the new measures: the ARL Lib-QUAL + Project-A Preliminary Report. Libraries and the Academy. pp. 103-112.

CRONIN, JOSEPH and STEVEN TAYLOR (1992). Measuring service quality: A reexamination and extension. Journal of Marketing. No. 56. pp. 55-68.

DONABEDIAN, AVEDIS (1984). La calidad de la atención médica. Definición y métodos de evaluación. La Prensa Médica Mexicana. México.

DUQUE, EDISON (2005). Revisión del concepto de calidad del servicio y sus modelos de medición. Innovar Revista de Ciencias Administrativas y Sociales. Vol. 15. No 25. pp. 64-80. Fuente: www.redalyc.org/pdf/818/81802505. pdf (Consultado 1411-14).

Donabedian, A. (s.f.). Universidad de Michigan. Obtenido de https://www.esan.edu.pe/apuntes-empresariales/2016/05/los-diferentesconceptos-de-calidad-en-salud/

El Comercio. (29 de septiembre de 2015), adipiscor.com. Obtenido de adipiscor.com: https://www.adipiscor.com/como/807/Afiliacion-al-IESS-de-las-Trabajadorasno-Remun

El Comercio. (26 de enero de 2016). https://www.adipiscor.com/como/807/Afiliacion-alIESS-de-las-Trabajadoras-no-Remun. Obtenido de adipiscor.com: https://www.adipiscor.com/como/807/Afiliacion-al-IESS-de-las-Trabajadorasno-Remun

Instituto Ecuatoriano de Seguridad Social. (2005). Obtenido de https://www.iess.gob.ec/es/inst-quienes-somos

Kotler, J. (2001).

Nacional, A. (5 de octubre de 2015). http://www.asambleanacional.gob.ec/es/contenido/afiliacion-de-las-trabajadorasno-remuneradas-del-hogar. Obtenido de asambleanacional.gob.ec: http://www.asambleanacional.gob.ec/es/contenido/afiliacion-de-las-trabajadorasno-remuneradas-del-hogar

Peralba, J. L. (2016). El es tu cliente o el mío. Argentina.

Piedra, J. (s.f.). Obtenido de encolombia: https://encolombia.com/economia/empresas/marcas/queesunproducto/

valverde, J. V. (11 de Mayo de 2016). Los diferentes conceptos de Calidad en la Salud. Obtenido de https://www.esan.edu.pe/apuntes-empresariales/2016/05/losdiferentes-conceptos-de-calidad-en-salud/

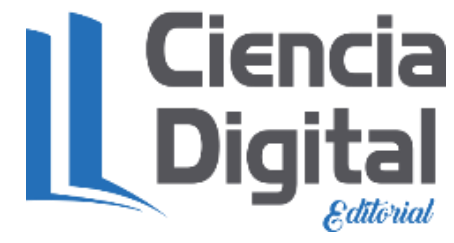




\section{Para citar el artículo indexado.}

Mayorga M., \& Gamboa J. (2019). Análisis de la ampliación de cobertura a la categoría de trabajadoras del hogar en la mejora de la calidad de servicio del afiliado al IESS del cantón Milagro del periodo 2015-17. Revista electrónica Visionario Digital 3(2), 126142.

http://www.cienciadigital.org/revistascienciadigital/index.php/VisionarioDigital/article/view/3 $\underline{99 / 893}$

\section{Ciencia \\ Ligital \\ Edtónial}

El artículo que se publica es de exclusiva responsabilidad de los autores y no necesariamente reflejan el pensamiento de la Revista Ciencia Digital.

El articulo queda en propiedad de la revista y, por tanto, su publicación parcial y/o total en otro medio tiene que ser autorizado por el director de la Revista Ciencia Digital.
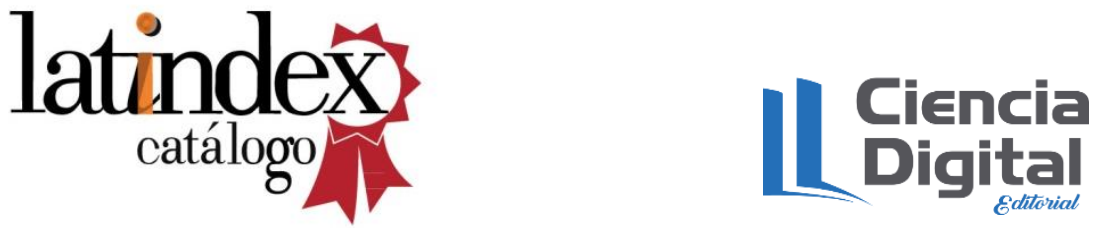